\title{
Nitrogen-regulated transcription and enzyme activities in continuous cultures of Saccharomyces cerevisiae
}

\author{
Eelko G. ter Schure, ${ }^{1}$ Herman H. W. Silljé, ${ }^{1}$ Leon J. R. M. Raeven, ${ }^{1}$ \\ Johannes Boonstra, ${ }^{1}$ Arie J. Verkleij ${ }^{1}$ and C. Theo Verrips ${ }^{1,2}$
}

Author for correspondence: Eelko G. ter Schure. Tel: +31 30 532598. Fax: +31 30513655.

\footnotetext{
1 Department of Molecular Cell Biology, Utrecht University, Padualaan 8, 3584 CH Utrecht, The Netherlands

2 Unilever Research Laboratorium Vlaardingen Olivier van Noortlaan 120, 3133 AT Vlaardingen, The Netherlands
}

\begin{abstract}
Variations in the transcription of nitrogen-regulated genes and in the activities of nitrogen-regulated enzymes of the yeast Saccharomyces cerevisiae were studied by changing the carbon and nitrogen fluxes. S. cerevisiae was grown in continuous culture at various dilution rates $(D)$ under nitrogen limitation with $\mathrm{NH}_{4} \mathrm{Cl}$ as sole nitrogen source. With an increase in $D$ from 0.05 to $0.29 \mathrm{~h}^{-1}$, both the glucose and the ammonia flux increased sixfold. The activities of the two ammonia-incorporating enzymes, NADPH-dependent glutamate dehydrogenase (NADPH-GDH) and glutamine synthetase (GS), encoded by GDH1 and GLN1, respectively, increased with increasing D, while the activity of the glutamate-degrading enzyme, NAD-dependent glutamate dehydrogenase (NAD-GDH), decreased. Surprisingly, no changes were observed in the transcription of GDH1 and GLN1; however increased D was accompanied by an increase in GAP1 transcription. At the metabolite level, the increase in the glucose and nitrogen flux did not result in changes in the intracellular 2oxoglutarate, glutamate or glutamine concentrations. It is shown that growth on ammonia alone is not sufficient to cause repression of GAP1 and GLN1 transcription and that the regulation of GAP1 transcription and both NADPHGDH and GS activity is not an on/off switch, but is gradually modulated in correlation with the ammonia concentration.
\end{abstract}

Keywords: Saccharomyces cerevisiae, nitrogen regulation, nitrogen limitation, nitrogen metabolism, continuous culture

\section{INTRODUCTION}

The main nutrients, with respect to quantity, used by the yeast Saccbaromyces cerevisiae are carbon and nitrogen. This implies that the carbon and nitrogen fluxes as well as their mutual interaction constitute important parameters in the regulation of cell growth. The coordination of the carbon and nitrogen fluxes may be regulated at the level of uptake or at the level of metabolism, depending upon the growth conditions and the nature of the carbon and nitrogen source. Glucose and ammonia can be used as sole carbon and nitrogen sources, respectively; their uptake is mediated both by specific low- and high-affinity permeases (Roon et al., 1977; Dubois \& Grenson, 1979; Bisson \& Fraenkel, 1983; Marini et al., 1994).

Abbreviations: $D$, dilution rate; GS, glutamine synthetase; NAD-GDH, NAD-dependent glutamate dehydrogenase; NADPH-GDH, NADPH-dependent glutamate dehydrogenase.
Intracellularly, carbon and nitrogen fluxes interact at the level of glutamate. During growth on ammonia as sole source of nitrogen, $\mathrm{NH}_{4}^{+}$is incorporated into 2-oxoglutarate by the NADPH-dependent glutamate dehydrogenase (NADPH-GDH) to produce glutamate. Subsequently, glutamate is converted to glutamine by the incorporation of another ammonium ion at the expense of ATP by glutamine synthetase (GS). On the other hand, during growth on glutamate as sole nitrogen source, glutamate is degraded to 2-oxoglutarate and $\mathrm{NH}_{4}^{+}$by the NADdependent glutamate dehydrogenase (NAD-GDH), to produce $\mathrm{NH}_{4}^{+}$for the synthesis of glutamine.

The regulation of the intracellular fluxes of carbon and nitrogen may be at substrate level or at transcription level. At substrate level it has been shown that glutamine exhibits strong allosteric inhibition of NAD-GDH and GS activity (Coschigano \& Magasanik, 1991). At transcriptional level the regulation is more complicated. The expression of the structural gene for NAD-GDH, GDH2, 
is regulated by the activity of the transcriptional activator Gln3p (Mitchell \& Magasanik, 1984a; Miller \& Magasanik, 1991). In the presence of ammonia or glutamine, the Gln $3 p$ activity is modulated by Ure2p. Ure2p causes an inactivation of $G \ln 3 p$ and therefore repression of GDH2 (Coschigano \& Magasanik, 1991). Also, the transcription of the structural gene for GS, GLN1, is regulated by $G \ln 3 p$ and consequently by Ure2p. Therefore Ure2p represses the transcription of both the GDH2 and the GLN1 gene by inhibiting the Gln3p activity. In addition the transcription of GLN1 is subject to general amino acid control, in contrast to the expression of GDH2. Prolonged amino acid starvation causes an increase in the transcription of GLN1 via an increased translation of the transcriptional activator Gcn4p (Dever et al., 1992). Besides GDH2 and GLN1 transcription, the transcription of other genes, e.g. GAP1 (encoding general amino acid permease), is also regulated by the $G \ln 3 p$ protein. Furthermore, Gln3p seems to be necessary for full gene expression of $G D H 1$, encoding NADPH-GDH, although $G D H 1$ transcription is supposed to be constant (Daugherty et al., 1993). A further illustration of the regulation between the carbon and nitrogen fluxes is the observation that the promotor region of $G D H 2$ contains a carbon-responsive upstream activation site in addition to a nitrogen-responsive element (Coschigano et al., 1991).

To gain insight into the intracellular coordination of the carbon and nitrogen fluxes, we have grown $S$. cerevisiae in a nitrogen-limited continuous culture. By using low carbon (glucose) concentrations under nitrogen (ammonia)-limiting conditions, the carbon flux and the nitrogen flux can be modulated by changing the dilution rate $(D)$. Ammonia was used because of its incorporation into carbon metabolites at well-defined steps. Firstly, the influence of the changes in the fluxes of nitrogen and carbon on the transcription of $G \ln 3 p$-regulated genes was analysed. Secondly, under the same conditions several intracellular metabolites, such as 2-oxoglutarate and amino acids, were analysed to study whether a correlation exists between the metabolite concentrations and the expression of $\mathrm{Gln} 3 \mathrm{p}$-regulated genes. It is demonstrated that variations within a narrow range of the carbon and nitrogen fluxes lead to changes in the activities and transcription of nitrogen-regulated enzymes and genes, respectively.

\section{METHODS}

Strain and growth conditions. S. cerevisiae strain SU32 was grown at $30^{\circ} \mathrm{C}$ in a 21 BioflollI fermenter (New Brunswick Scientific) connected to a computer controller unit running with Advanced Fermentation Software (New Brunswick Scientific). S. cerevisiae SU32 was inoculated in a medium previously described by Sierkstra et al. (1992), and after overnight growth a continuous feed was connected. Subsequently the yeast was grown at $D$ of $0 \cdot 05,0 \cdot 1,0 \cdot 15,0 \cdot 19$ and $0.29 \mathrm{~h}^{-1}$ with $1.5 \mathrm{~g} \mathrm{NH}_{4} \mathrm{Cl}$ $\mathrm{I}^{-1}$ and $9.5 \mathrm{~g}$ glucose $\mathrm{l}^{-1}$ in the feed. During batch and nitrogenlimited growth the $\mathrm{pH}$ was automatically controlled at 5 by the addition of $5 \mathrm{M} \mathrm{KOH}$. The airflow was $1.51 \mathrm{~min}^{-1}$ and the oxygen tension was kept above $50 \%$ by controlling the stirrer speed. Carbon dioxide production $\left[r_{\mathrm{CO}_{2}}\right.$ in $\mathrm{mmol}(\mathrm{g}$ dry $\left.\mathrm{wt})^{-1} \mathrm{~h}^{-1}\right]$ and oxygen consumption $\left[q_{\mathrm{o}_{2}}\right.$ in $\left.\mathrm{mmol} \mathrm{g} \mathrm{g}^{-1} \mathrm{~h}^{-1}\right]$ were measured on-line by connection of the fermenter headspace to an Uras3G $\mathrm{CO}_{2}$ analyser and a Magnos4G $\mathrm{O}_{2}$ analyser (Hartmann \& Braun).

Sampling and preparation of samples. When steady state was reached, samples were taken in duplicate for three successive days, and immediately frozen in liquid nitrogen and stored at $-80^{\circ} \mathrm{C}$. The total sample volume was approximately $50 \mathrm{ml}$. Preparation of cell-free extracts for metabolite determination, enzyme assays, mRNA isolation and dry weight determination were performed as described by Sierkstra et al. (1992). Free amino acids were isolated by resuspending $3 \mathrm{ml}$ frozen culture sample into $15 \mathrm{ml}$ boiling ethanol. The samples were boiled for $5 \mathrm{~min}$ and concentrated threefold by evaporation of approximately $12 \mathrm{ml}$ suspension in a vacuum dryer. Sampling of the culture medium for the analyses of extracellular metabolites (acetate, glycerol, ethanol, glucose and $\mathrm{NH}_{4}^{+}$concentrations) was performed by quick filtration (within $10 \mathrm{~s}$ after sampling) of the culture liquid through a $0.22 \mu \mathrm{m}$ membrane filter.

Determination of metabolites and free amino acids. Extracellular metabolites were measured by means of HPLC analysis or enzymically, essentially as described by Bergmeyer (1974). All metabolites of glycolysis and the citric acid cycle were measured spectrophotometrically as described previously by Sierkstra et al. (1992) using a Hitachi U2000 spectrophotometer. All values are given as an average of three independent samples \pm SEM. Free amino acid analysis was performed on an ALPHA PLUS amino acid analyser, series 2 (Pharmacia LKB). Glutamate concentrations were also measured using the L-glutamic acid determination kit (Boehringer Mannheim).

Labelling of oligonucleotides and Northern blot analysis. For the detection of the $A C T 1$ and $H 2 A / H 2 B$ mRNA, labelled oligonucleotides were used as described by Sierkstra et al. (1992). The other oligonucleotides used for the detection of mRNAs in Northern analyses were: $5^{\prime}$-CAGCGGAGCCATTTGAGGGAATGGTTATTG-3' (GAP1); 5'-TGTAGTGAGCTTCGATCATGTCTCTGGCAT-3' (GLN1); 5'-AAAGAGGAGACAACTTCTTCGTAAGCTTGTTGAAATTCTGGCTCTGACAT-3' (GDH1); 5'-GATCAATCTGGCGGCTTGAGTTCTCAACAT-3' (ILV5); and 5'-ACCAGCTAGAACAATCTTGGAAGCGCCAAC-3' (HIS4). Northern analyses were performed as described by Sierkstra et al. (1992). Northern blots for detection of the GAP1, HIS4 and ILV 5 mRNA levels were probed with $A C T 1$ as internal control for the amount of RNA blotted, for the detection of GLN1 and $G D H 1$ mRNA levels, $H 2 A / H 2 B$ was used as an internal control.

Enzyme assays. The activities of NADPH-GDH and NADGDH were measured under $V_{\max }$ conditions essentially as described by Boles et al. (1993). GS activity was measured as described by Mitchell \& Magasanik (1983). Reaction velocities were proportional to the amount of enzyme added. The protein content of the cell extracts was determined by the method of Petterson (1977).

\section{RESULTS}

\section{Growth conditions and cell morphology}

S. cerevisiae SU32 was grown in nitrogen-limited continuous culture using ammonia as sole nitrogen source and glucose as sole carbon source. To determine the minimum medium concentrations of glucose and ammonia at which the culture was growing nitrogen-limited, S. cerevisiae was grown at $D 0 \cdot 1 \mathrm{~h}^{-1}$ with a feed containing $1.5 \mathrm{~g} \mathrm{NH}_{4} \mathrm{Cl} \mathrm{l}^{-1}$ and increasing glucose concentrations, 


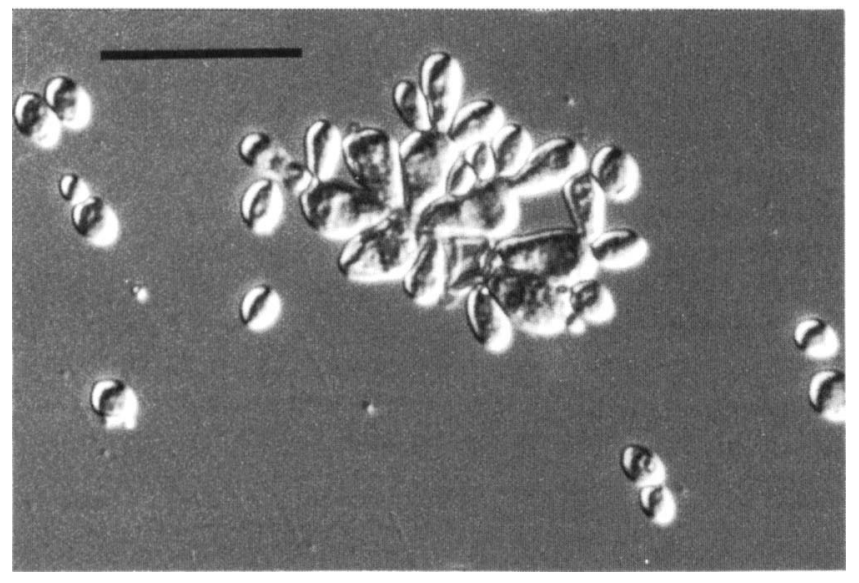

Fig. 1. Morphology of pseudohyphae of $S$. cerevisiae in a nitrogen-limited continuous culture. Bar, $20 \mu \mathrm{m}$.

starting at $7.0 \mathrm{~g} \mathrm{l}^{-1}$. At glucose concentrations higher than $8.5 \mathrm{~g} \mathrm{l}^{-1}$ no further increase in the biomass was observed (data not shown), indicating that with $1.5 \mathrm{~g}$ $\mathrm{NH}_{4} \mathrm{Cl} \mathrm{l}^{-1}$ and $8.5 \mathrm{~g}$ glucose $\mathrm{l}^{-1}$ in the feed, the culture was nitrogen-limited. To ensure nitrogen limitation the glucose concentration used was $9 \cdot 5 \mathrm{~g} \mathrm{l}^{-1}$ and subsequently the yeast was grown at $D$ values of $0 \cdot 05,0 \cdot 1,0 \cdot 15,0 \cdot 19$ and $0.29 \mathrm{~h}^{-1}$. The cells exhibited a pseudohyphal-like morphology at all growth rates (Fig. 1). Gimeno et al. (1992) described a similar morphology of $S$. cerevisiae when growing on nitrogen-limited agar plates. Therefore, these data indicate that $S$. cerevisiae was nitrogen-limited under the conditions employed.

\section{Physiological parameters}

To be able to correlate the expression of genes involved in nitrogen and carbon metabolism to the fluxes of nitrogen and carbon, a number of physiological parameters were determined to establish the growth conditions of the yeast in detail. As shown in Fig. 2(a), with an increase in $D$ from 0.05 to $0.29 \mathrm{~h}^{-1}$, the specific $\mathrm{CO}_{2}$ production increased sixfold from 1.6 to $9.8 \mathrm{mmol} \mathrm{g}^{-1} \mathrm{~h}^{-1} ; \mathrm{O}_{2}$ consumption also increased sixfold, from 1.5 to $9 \cdot 0 \mathrm{mmol} \mathrm{g}^{-1} \mathrm{~h}^{-1}$. Consequently the respiration quotient was constant at around $1 \cdot 1$ at all $D$ values. This indicates that no major changes occurred in the carbon metabolism under these conditions, a finding which is supported by the observation that no ethanol, glycerol or acetate could be detected in the medium (detection limits $0.05 \mathrm{~g} \mathrm{l}^{-1}$ ) (data not shown). The biomass remained constant at about $4.4 \mathrm{~g}^{-1}$ (Fig. 2b). As shown in Fig. 2(c), the residual
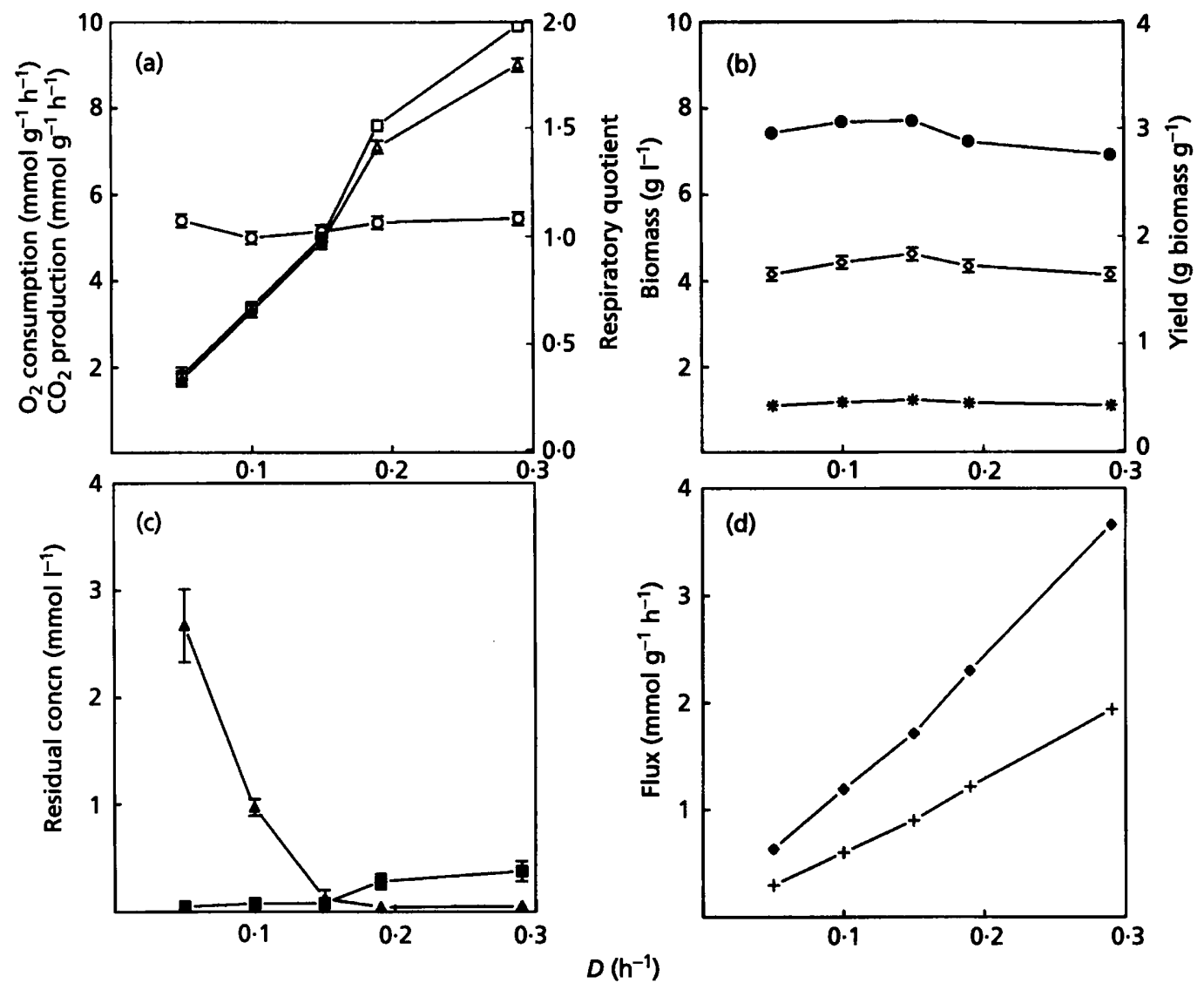

Fig. 2. (a) Production of $\mathrm{CO}_{2}(\square)$, consumption of $\mathrm{O}_{2}(\triangle)$ and repiratory quotient $(O)$; (b) biomass $(\diamond)$, yield on glucose $(*)$ and on ammonia $(O)$; (c) residual concentrations of glucose $(\square)$ and ammonia $(A) ;(d)$ flux of glucose $(\bullet)$ and of ammonia $(+)$ into the cells. All values are given as the mean of three independent samples \pm SEM. 

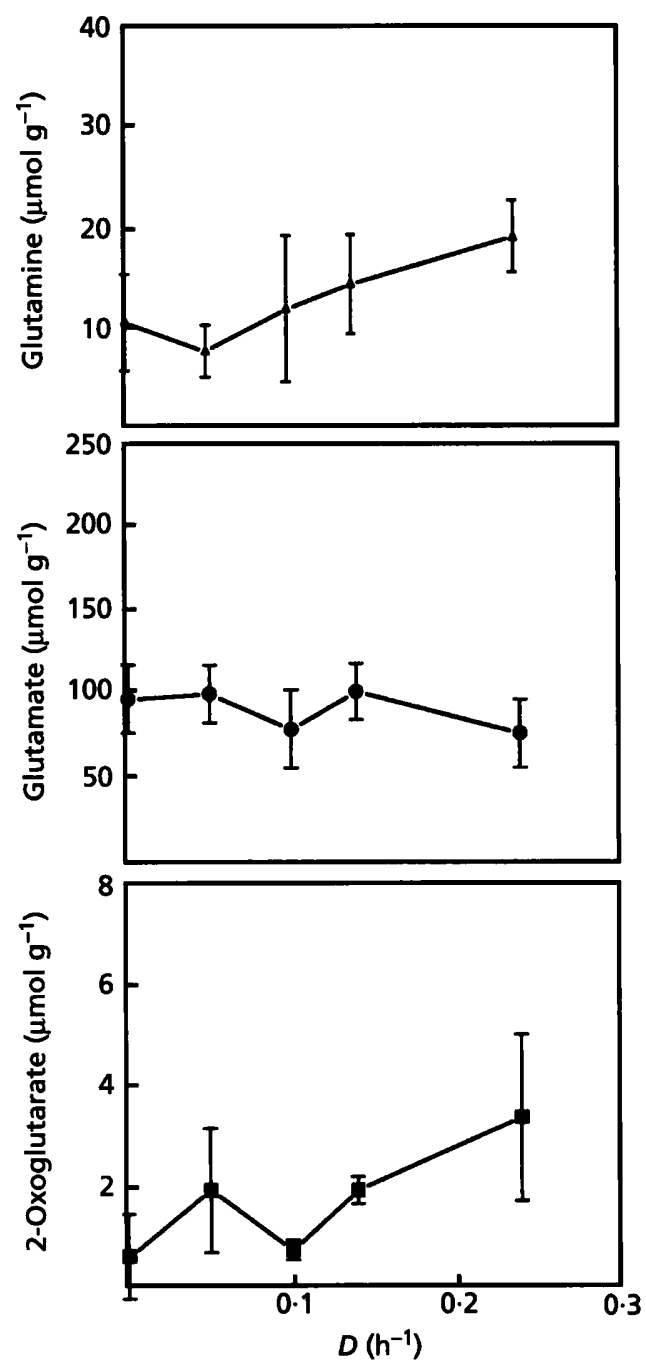

Fig. 3. Concentrations of glutamine, glutamate and 2oxoglutarate in a nitrogen-limited continous culture at different $D$ values. All values are given as the mean of three independent samples \pm SEM.

$\mathrm{NH}_{4}^{+}$concentration was $2.7 \mathrm{mM}$ at $D 0.05 \mathrm{~h}^{-1}$, and decreased rapidly to $1.0 \mathrm{mM}$ at $D 0.1 \mathrm{~h}^{-1}$, followed by a more gradual decrease to $0.05 \mathrm{mM}$ at $D 0.29 \mathrm{~h}^{-1}$. The nitrogen and carbon fluxes were determined respectively from the residual ammonia and glucose concentration in the medium in combination with the $D$ value, and as shown in Fig. 2(d), the nitrogen flux increased linearly approximately sixfold with increasing $D$ from 0.05 to $0.29 \mathrm{~h}^{-1}$. In contrast to the residual ammonia concentration, the residual glucose concentration increased from 0.01 to $0.4 \mathrm{mM}$ with an increase in $D$ from 0.05 to $0.29 \mathrm{~h}^{-1}$ (Fig. 2c). Nevertheless the glucose flux calculated from these data also increased linearly approximately sixfold with an increase in $D$ from 0.05 to $0.29 \mathrm{~h}^{-1}$ (Fig. 2d). Because the residual concentrations of ammonia and glucose are small compared to their concentrations in the feed $(<1 \%)$, these variations do not significantly influence the respective fluxes.
Table 1. Activities of NADPH-GDH, NAD-GDH and GS in proline-, glutamate- and glutamine-grown batch cultures

\begin{tabular}{|llll|}
\hline Nitrogen source & \multicolumn{3}{c|}{ Specific activity* of: } \\
\cline { 2 - 4 } & $\begin{array}{c}\text { NADPH- } \\
\text { GDH }\end{array}$ & NAD-GDH & GS \\
& & \\
\hline Proline & $1.23 \pm 0.06$ & $0.018 \pm 0.001$ & $0.93 \pm 0.17$ \\
Glutamate & $1.37 \pm 0.03$ & $0.550 \pm 0.016$ & $1.65 \pm 0.16$ \\
Glutamine & $0.97 \pm 0.03$ & $0.006 \pm 0.003$ & $0.14 \pm 0.02$ \\
\hline
\end{tabular}

* Specific activity in $\mu \mathrm{mol}$ substrate $\mathrm{min}^{-1}$ (mg total soluble cellular protein $)^{-1}$. Values are given as the mean of three independent samples \pm SEM.

Because both the ammonia and glucose consumption increased linearly with $D$ and no changes in the biomass were observed, the yields on ammonia and glucose in the whole range of $D$ values remained constant at about

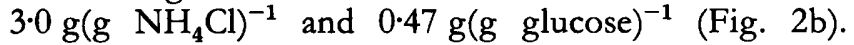
Altogether, the data presented clearly demonstrate that, under the conditions employed, the fluxes of both ammonia and glucose increased linearly with $D$ and no changes occurred in the carbon metabolism.

\section{Levels of glycolytic metabolites}

Although the physiological parameters indicated no major changes in the carbon metabolism, the levels of glycolytic intermediates and ATP were determined. The glucose uptake was shown to increase linearly with $D$ and consequently the glycolytic flux also increased with $D$. With increasing $D$ the levels of the glycolytic intermediates glucose 6-phosphate, fructose 6-phosphate and fructose 1,6-diphosphate remained constant at $3 \cdot 19 \pm 0 \cdot 25$, $0.51 \pm 0.05$ and $4.52 \pm 0.39 \mu \mathrm{mol} \mathrm{g}^{-1}$, respectively. The ATP concentration also did not change significantly and remained at $4 \cdot 25 \pm 0 \cdot 60 \mu \mathrm{mol} \mathrm{g}^{-1}$.

\section{2-Oxoglutarate and amino acid concentrations}

2-Oxoglutarate, glutamate and glutamine play an essential role in the interaction between carbon and nitrogen metabolism. Therefore, their respective concentrations were analysed to investigate if changes in these concentrations could be linked to the regulation of the nitrogen metabolism. As is shown in Fig. 3 the 2-oxoglutarate, glutamine and glutamate concentrations did not change significantly and remained at about 5, 27 and $180 \mu \mathrm{mol} \mathrm{g}^{-1}$, respectively.

\section{Expression of nitrogen-regulated genes}

To investigate the regulation of gene expression as a function of the nitrogen and carbon fluxes, mRNA was isolated from cells grown at various $D$ values, and the expression of various genes was determined by Northern blotting. These genes included GDH1 (encoding 

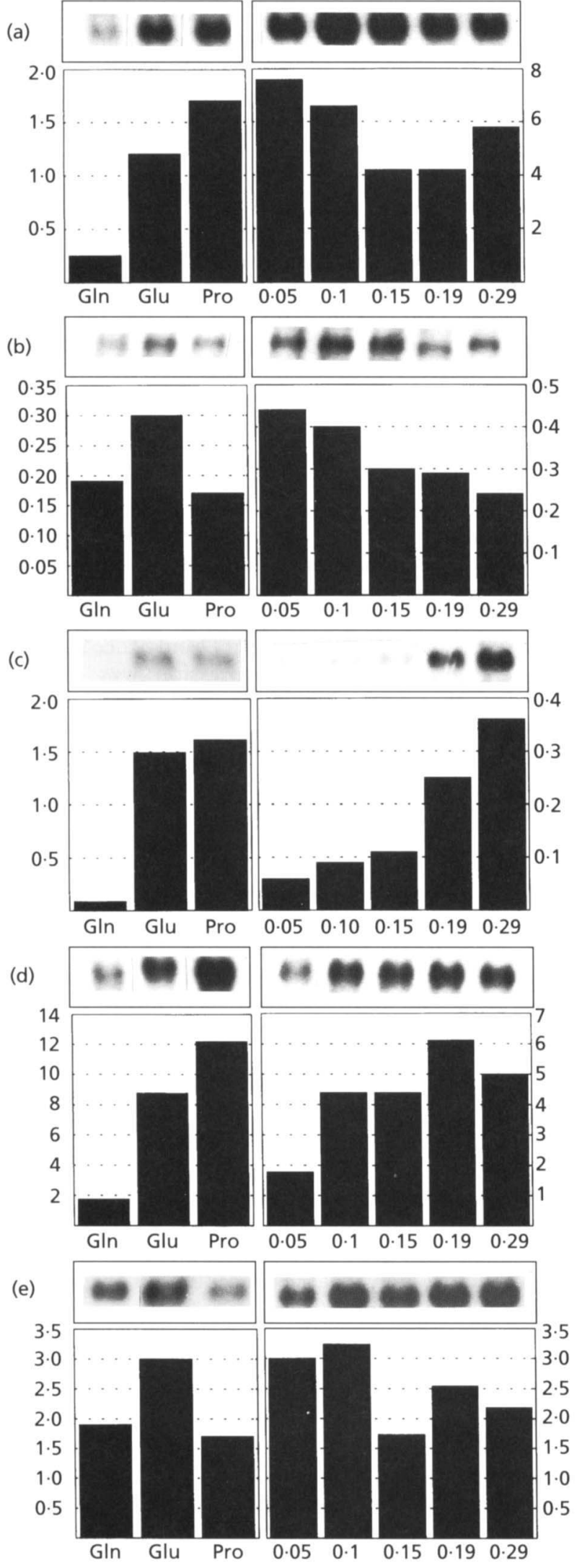

Fig. 4. mRNA levels from the GDH1 (a), HIS4 (b), GAP1 (c), GLN1 (d) and ILV5 (e) genes. The levels of the GAPI, ILV5 and HIS4 transcripts were compared with $A C T 1$ as an internal control,
NADPH-GDH), GLN1 (encoding GS), GAP1 (encoding general amino acid permease), ILV 5 (encoding $\alpha$ acetolactate reductoisomerase) and HIS4 (encoding histidinol dehydrogenase). The transcription of $G A P 1$ was used as a marker for Gln3p activity. ILV 5 and HIS4 are both under the control of the Gen $4 p$ transcriptional activator.

Expression of these genes was analysed in batch cultures grown on glutamine, glutamate and proline as the sole nitrogen source to compare these data with those determined from the continuous culture experiments. In agreement with previously presented data (Benjamin $e t$ al., 1989; Jauniaux \& Grenson, 1990; Minehart \& Magasanik, 1992; Daugherty et al., 1993), expression of the GAP1, GLN1 and GDH1 was repressed during growth on glutamine and derepressed during growth on proline and glutamate, while no differences in the expression of the ILV5 and HIS4 genes were observed during growth on the various nitrogen sources. (Fig. 4).

In a nitrogen-limited continuous culture with a small excess of glucose, the amount of GAP1 mRNA increased with $D$ (Fig. 4). The increase of GAP1 transcription correlates with the increase in the nitrogen and carbon fluxes, as well as the decrease in the extracellular ammonia concentration. The level of GLN1, GDH1, ILV 5 and HIS4 mRNAs did not change with increasing D. From these data we conclude that growth on ammonia alone is not sufficient to cause repression of GAP1 and GLN1 gene expression.

\section{Enzyme activities}

Glucose and ammonia fluxes increased linearly with $D$. To investigate whether the yeast reacted to the increase of these fluxes by increasing the amount or activities of the enzymes involved in the incorporation of ammonia into 2-oxoglutarate and glutamate, the maximum activities of these enzymes were analysed in crude extracts.

First their activities were determined in glutamine-, glutamate- and proline-grown batch cultures to compare these data with those found in continuous culture. In proline- and glutamate-grown cultures, the activity of NADPH-GDH was slightly higher than in glutaminegrown cultures (Table 1). As has been shown previously (Mitchell \& Magasanik, 1984a), the activities of both NAD-GDH and GS were higher during growth on proline and glutamate than during growth on glutamine.

and the levels of the GDH1 and GLN1 transcripts were compared with H2A/2B. Northern blots are shown in the upper panels. The data were quantified by calculating the intensity ratio between the gene of interest and the reference gene; these ratios are shown in the lower panels. Transcription levels in batch-grown cultures with glutamine (Gln), glutamate (Glu) or proline (Pro) as the sole nitrogen source are shown in the left panels. Transcription levels in nitrogen-limited continuous cultures at $D 0.05,0.1,0.15,0.19$ and $0.29 \mathrm{~h}^{-1}$ are shown in the right panels. 
Table 2. Activities of NADPH-GDH, NAD-GDH and GS in an ammonia-limited continuous culture at different $D$ values

\begin{tabular}{|lccc|}
\hline $\boldsymbol{D}$ & \multicolumn{3}{c|}{ Specific activity* of: } \\
\cline { 2 - 4 }$\left(\mathbf{h}^{-1}\right)$ & NADPH-GDH & NAD-GDH & GS \\
\hline 0.05 & $1.10 \pm 0.03$ & $0.033 \pm 0.006$ & $0.36 \pm 0.07$ \\
0.1 & $1.45 \pm 0.06$ & $0.043 \pm 0.004$ & $0.52 \pm 0.02$ \\
0.15 & $1.91 \pm 0.42$ & $0.042 \pm 0.008$ & $0.72 \pm 0.11$ \\
0.19 & $2.24 \pm 0.32$ & $0.023 \pm 0.001$ & $0.90 \pm 0.10$ \\
0.29 & $2.03 \pm 0.16$ & $0.012 \pm 0.002$ & $1.02 \pm 0.09$ \\
\hline
\end{tabular}

*Specific activity in $\mu \mathrm{mol}$ substrate $\mathrm{min}^{-1}$ ( $\mathrm{mg}$ total soluble cellular protein $)^{-1}$. Values are given as the mean of three independent samples \pm sEM.

As shown in Table 2, in a nitrogen-limited continuous culture with a small excess of glucose, the activity of the NADPH-GDH increased from 1.10 to $2.03 \mu \mathrm{mol} \mathrm{min}^{-1}$ $(\mathrm{mg} \text { protein })^{-1}$, and the activity of GS increased from 0.36 to $1.02 \mu \mathrm{mol} \mathrm{min}^{-1}$ (mg protein) ${ }^{-1}$, when $D$ was increased from 0.05 to $0.29 \mathrm{~h}^{-1}$; the NAD-GDH activity decreased from 0.033 to $0.012 \mu \mathrm{mol} \mathrm{min}^{-1}$ (mg protein $)^{-1}$. Because the mRNA level of the GDH1 and GLN1 genes did not change by the same amount as the activities of NADPH-GDH and GS we conclude that the enzymes are also regulated at a post-transcriptional level. Comparison with proline-, glutamate- and glutaminegrown batch cultures reveals that the NADPH-GDH and GS activities at lower $D$ are similar to glutamine-grown cultures, and at higher $D$ are similar to proline- or glutamate-grown cultures.

\section{DISCUSSION}

An ammonia-limited continuous culture of $S$. cerevisiae with a small excess of glucose was used to investigate the influence of the carbon and nitrogen fluxes on the regulation of transcription and activities of enzymes involved in the interaction of these fluxes. By varying the concentration of glucose in the feed it was determined

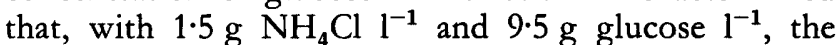
culture was nitrogen-limited at $D 0.1 \mathrm{~h}^{-1}$ (data not shown). The pseudohyphal-like morphology also indicated that the culture was nitrogen-limited at all other $D$ values.

Both the ammonia (nitrogen) and glucose (carbon) flux increased linearly with $D$, and no significant changes in the carbon metabolism occurred, as can be concluded from the observation that the increase in glucose uptake was equal to the increase in $\mathrm{CO}_{2}$ production and $\mathrm{O}_{2}$ consumption, and that no ethanol, glycerol or acetate were detected in the culture medium. Nevertheless, significant changes were observed in the residual concentrations of ammonia and glucose. With increasing $D$ the residual ammonia concentration decreased and the residual glucose concentration increased. An increase in the carbon and nitrogen flux did not result in changes in the concentrations of glycolytic intermediates, 2-oxoglutarate, glutamate or glutamine, which was in line with the linear increase in both the glucose and ammonia flux with $D$.

The expression of genes encoding nitrogen-regulated enzymes and the activity of these enzymes is known to be dependent upon the nature of the nitrogen source used by the yeast (Mitchell \& Magasanik, 1984b; Miller \& Magasanik, 1991). The expression of the GLN1 and GAP1 genes is low during growth on a repressible nitrogen source, e.g. glutamine, and high during growth on a derepressible nitrogen source, e.g. proline. These differences in mRNA expression are caused by an altered activity of the transcriptional activator $G \ln 3 p$ (Mitchell \& Magasanik 1984a). Except for the fact that full expression of the GDH1 gene is dependent upon the presence of the GLN3 gene product (Daugherty et al., 1993), not much is known about its transcriptional regulation. The influence of the nitrogen and carbon flux on the regulation of the GDH1 and GLN1 gene expression was analysed by mRNA detection. With increasing $D$ and hence increasing fluxes, no change in their expression was observed. However, the expression of another $G \ln 3 p$-regulated gene, $G A P 1$, increased with $D$, indicating increased activity of the transcriptional activator $\mathrm{Gln} 3 \mathrm{p}$. In contrast to $G A P 1$, the expression of $G L N 1$ is also regulated by the transcriptional activator Gcn4p. The expression of two other Gcn4p-regulated genes, ILV 5 and HIS4, was constant at all $D$ values, indicating no change in the activity of Gcn4p. Therefore the constant expression of GLN1 can be explained by the constant activity of Gcn4p which overrides the increased activity of $G \ln 3 p$. Alternatively, the increase in GAP1 mRNA levels might also be caused by a $G \ln 3 p$-independent mechanism, e.g. a decreased activity of a PER1-regulated pathway (Courchesne \& Magasanik, 1983), caused by the lower residual ammonia concentrations at higher $D$.

From the increased GAP1 expression at high $D$ it can be concluded that the use of ammonia as nitrogen source alone is insufficient to cause repression of the GAP1 transcription; moreover, no change in the concentrations of glutamate and glutamine could be observed, and it is therefore more likely that the concentration of ammonia is causing the repression.

With increasing $D$ and thus decreasing residual $\mathrm{NH}_{4}^{+}$ concentrations, the activities of the enzymes using $\mathrm{NH}_{4}^{+}$ for the synthesis of glutamate and glutamine, NADPHGDH and GS, respectively, increased whereas the activity of the glutamate-degrading and $\mathrm{NH}_{4}^{+}$-producing enzyme, NAD-GDH, decreased with increasing $D$. At low $D$ the NADPH-GDH and GS activities were comparable to glutamine-grown batch cultures. At higher $D$, however, their activities became similar to proline- or glutamategrown batch cultures. The increased activity of NADPHGDH and GS is not caused by elevated transcription, as shown by constant $G D H 1$ and GLN1 mRNA levels, and 


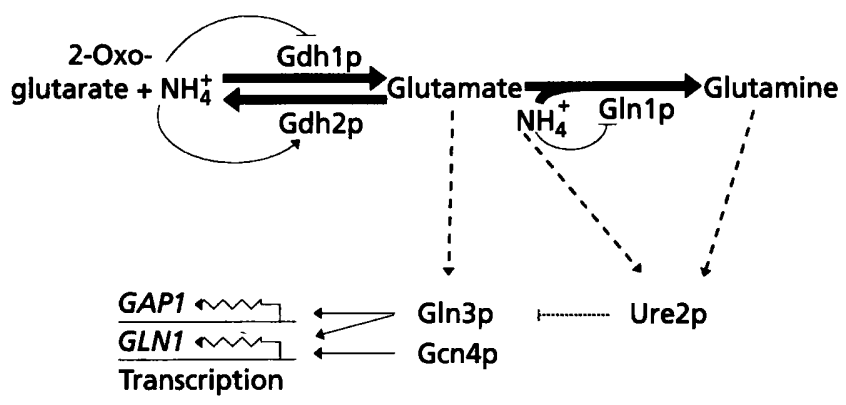

Fig. 5. Regulation of GAP1 and GLN1 transcription and NADPHGDH, NAD-GDH and GS activities in response to the concentration of ammonia. Enzymic reactions are indicated by bold arrows. Regulations are indicated by thin arrows, activation is indicated by $\rightarrow$, and inhibition is indicated by $\dashv$. Activiation of Ure2p and Gln3p by glutamine, ammonia or glutamate is indicated by dashed arrows.

must therefore be caused by a translational or an allosteric mechanism.

These data demonstrate that when $D$ is increased the carbon and the nitrogen fluxes increase, the GAP1 mRNA expression increases and the GDH1 and GLN1 mRNA expression remain constant but the activities of their gene products increase. These changes in mRNA expression and enzyme activities may be caused by the decreased extracellular ammonia concentration. This could be accomplished by reduced allosteric inhibition of the enzymes directly or via allosteric control of a regulator, e.g. Ure2p. In the latter case the ammonia concentration can decrease the Ure2p activity, which in turn regulates transcription via modulation of the Gln $3 p$ activity and also regulates enzyme activity directly. These regulation mechanisms at the level of activity and transcription are described in Fig. 5.

In conclusion, it is shown that with increasing $D$ in an ammonia-limited continuous culture, the activities of the nitrogen-regulated gene, $G A P 1$, and the nitrogen-regulated enzymes NADPH-GDH, NAD-GDH and GS, are modulated gradually. Moreover, it is shown that these modulations do correlate with the extracellular ammonia concentration and not with the intracellular glutamine or glutamate concentrations. Additional experiments are being performed to investigate the relationship between the $\mathrm{NH}_{4}^{+}$concentration and the enzyme and gene activities in more detail.

\section{ACKNOWLEDGEMENTS}

The authors would like to thank B. Bes for the HPLC analysis and M. Suijker for the free amino acid analysis.

\section{REFERENCES}

Benjamin, P. M., Wu J.-L., Mitchell, A. P. \& Magasanik B. (1989). Three regulatory systems control expression of glutamine synthetase in Saccharomyces cerevisiae. Mol \& Gen Genet 217, 370-377.
Bergmeyer, H. U. (1974). Methods of Enzymatic Analysis. Weinheim: Verlag Chemie.

Bisson, L. F. \& Fraenkel, D. G. (1983). Involvement of kinases in glucose and fructose uptake by Saccharomyces cerevisiae. Proc Natl Acad Sci US A 80, 1730-1734.

Boles, E., Lehnert, W. \& Zimmermann, F. K. (1993). The role of the NAD-dependent glutamate dehydrogenase in restoring growth of a Saccharomyces cerevisiae phosphoglucose isomerase mutant. Eur J Biochem 217, 469-477.

Coschigano, P. W. \& Magasanik, B. (1991). The Ure2 gene product of Saccharomyces cerevisiae plays an important role in the cellular response to the nitrogen source and has homology to glutathione $S$ transferases. Mol Cell Biol 11, 822-832.

Coschigano, P. W., Miller, S. M. \& Magasanik, B. (1991). Physiological and genetic analysis of the carbon regulation of the NADdependent glutamate dehydrogenase of Saccharomyces cerevisiae. Mol Cell Biol 11, 4455-4465.

Courchesne, W. E. \& Magasanik, B. (1983). Ammonia regulation of amino acid permeases in Saccharomyces cerevisiae. Mol Cell Biol 3, 672-683.

Dever, T. E., Feng, L., Weh, R. C., Cigan, A. M., Donahue, T. F \& Hinnebusch, A. G. (1992). Phosphorylation of initiation factor $2 \alpha$ by protein GCN2 mediates gene specific translational control of GCN4 in yeast. Cell 68, 585-596.

Daugherty, J. R., Rai, R., El Berry, H. M. \& Cooper, T. G. (1993). Regulatory circuit for responses of nitrogen catabolic gene expression to the GLN3 and DAL80 proteins and nitrogen catabolite repression in Saccbaromyces cerevisiae. $J$ Bacteriol 175, 64-73.

Dubois, E. \& Grenson, M. (1979). Methylamine/ammonia uptake systems in Saccharomyces cerevisiae: multiplicity and regulation. Mol \& Gen Genet 175, 67-76.

Gimeno, C. J., Ljundahl, P. O., Styles, C. A. \& Fink, G. R. (1992). Unipolar cell divisions in the yeast Saccharomyces cerevisiae lead to filamentous growth: regulation by starvation and ras. Cell 68, 1077-1090.

Jauniaux, J.-C. \& Grenson M. (1990). GAP1, the general amino acid permease gene of Saccharomyces cerevisiae. Nucleotide sequence, protein similarity with other bakers yeast amino acid permeases, and nitrogen catabolite repression. Eur J Biochem 190, 39-44.

Marini, A.-M., Vissers, S., Urrestarazu, A. \& André, B. (1994). Cloning and expression of the MEP1 gene encoding an ammonium transporter in Saccharomyces cerevisiae. EMBO J 13, 3456-3463.

Miller, S. M. \& Magasanik, B. (1991). Role of the complex upstream region of the GDH2 gene in nitrogen regulation of the NADlinked glutamate dehydrogenase in Saccharomyces cerevisiae. Mol Cell Biol 11, 6229-6247.

Minehart, P. L. \& Magasanik, B. (1992). Sequence of the GLN1 gene of Saccbaromyces cerevisiae: role of the upstream region in regulation of glutamine synthetase expression. J Bacteriol 174, 1828-1836.

Mitchell, A. P. (1985). The GLN1 locus of Saccharomyces cerevisiae encodes glutamine synthetase. Genetics 111, 243-258.

Mitchell, A. P. \& Magasanik, B. (1983). Purification and properties of glutamine synthetase from Saccharomyces cerevisiae. J Biol Chem 258, 119-124.

Mitchell, A. P. \& Magasanik, B. (1984a). Regulation of glutamine repressible gene products by the GLN3 function in Saccharomyces cerevisiae. Mol Cell Biol 4, 2758-2766.

Mitchell, A. P. \& Magasanik, B. (1984b). Three regulatory systems 
control production of glutamine synthetase. Mol Cell Biol 4, 2767-2773.

Petterson, G. L. (1977). A simplification of the protein assay method of Lowry et al., which is more generally applicable. Anal Biochem 83, 346-356.

Roon, R. J., Meyer, G. M. \& Larimore, F. S. (1977). Evidence for a common component in kinetically distinct transport systems of Saccharomyces cerevisiae. Mol \& Gen Genet 158, 185-191.
Sierkstra, L. N., Verbakel, J. M. A. \& Verrips C. T. (1992). Analysis of transcription and translation of glycolytic enzymes in glucoselimited continuous cultures of Saccharomyces cerevisiae. J Gen Microbiol 138, 2559-2566.

Received 5 August 1994; revised 22 December 1994; accepted 9 January 1995. 